\title{
Accounting for the surface temperature persistence by using signal energy
}

\author{
Jiangnan $\mathrm{Li}^{1}$ (D) $\cdot$ Zhian Sun $^{2} \cdot$ Feng Zhang ${ }^{3}$
}

Received: 9 March 2020 / Accepted: 6 December 2020 / Published online: 9 February 2021

(C) The Author(s) 2021

\begin{abstract}
The autocorrelation function (ACF) and its finite Fourier transform, referred to as signal energy, have been investigated using the ECMWF daily surface temperature data. ACF itself provides a measure of the influence of leading fluctuation between two different time points. Considering the decay of ACF, it is found that the scaling power-rule of ACF is only valid in a very short period, as the decay of ACF exists before it reaches a random noise state. Therefore, the method of the critical exponent of ACF is limited in the short length of the temporal interval. On the other hand, the distributions of the signal energy always show nice patterns, indicating the degree of persistence change. It is found, for a short period, that the distributions of the signal energy and the critical exponent are very similar, with a correlation coefficient over 0.97. For a longer period, though the critical exponent of ACF becomes invalid, the signal energy can always provide an effective method to investigate climate persistence in different lengths of time. In a 5-day period of boreal winter, the southern part of North America has a larger value of signal energy compared to the northern part; thus, the surface temperature is more stable in the north part. The result becomes opposite in the boreal summer. The method of signal energy can also be applied to a particular interval of time. In different temporal intervals, the signal energy presents very different results, especially over the El Nino regions
\end{abstract}

\section{Introduction}

The autocorrelation function (ACF), written as $C(\tau)$, provides a measure of the influence of leading fluctuations at the origin time $t$ on the behavior at over a temporal interval of $\tau$. The statistical average of the correlation between two temporal points is fundamental for understanding correlations in climate (Tsonis et al. 1999; Talkner and Weber 2000; Weber and Talkner 2001; Kantelhardt et al. 2001; Vyushin and Kushner 2009; Monettia et al. 2003; Lin et al. 2007; Monahan 2012; Rypdal et al. 2013; Yuan et al. 2015; Zhao and He 2015; He et al. 2016; Li and Sun 2020). The ACF itself can be used to measure directly the correlation

Jiangnan Li

jiangnan.li@canada.ca

1 Canadian Centre for Climate Modelling and Analysis, Environment and Climate Change Canada, Victoria, British Columbia, Canada

2 Research Program, Australian Bureau of Meteorology, Melbourne, Victoria, Australia

3 Institute of Atmospheric Sciences, Fudan University, Shanghai, China for the leading fluctuation between any two distinct time points with a temporal interval apart. The strength of the correlation of $\mathrm{AC}$ indicates the persistence (or recurrence) in climate events.

The values of ACF generally decrease with the increase of the temporal intervals; thus, ACF always implies a trend of decay. An interesting question is: what kind of rule does the decay behavior of ACF obey, and should the decay of ACF follow the power-law? This question has not been fully understood yet (Koyama and Hara 1992). Generally, ACF does not show the power-law decay, but exhibits multiple types of decay performances with different $\tau$. When the temporal interval becomes large enough, ACF always shows characteristics of random noise, as the two far away temporal moments are not correlated at all (Kantelhardt et al. 2001).

The Fourier transform of ACF has been widely used to analyze the random signal in many fields, and the power spectral density extracted from ACF can describe the variations that $\mathrm{ACF}$ distributes among the various frequencies. The power spectral density, which corresponds to the signal energy, is essentially the square of the magnitude of the Fourier transform. Parseval's theorem shows that the total power in the time domain is the same as the total power 
in the frequency domain. However, in the frequency space the signal processing can be resolved more precisely.

For a finite length of data, the Fourier transform of ACF becomes discrete in digital. For a finite temporal domain, there is a basic frequency dependent on the length of the domain. All other frequencies are the integer multiple of the basic frequency, and the number of frequency is limited. The summation of finite Fourier transform of ACF with all possible frequencies is called signal energy. Using the signal energy, the variation of ACF in different temporal domains can be resolved differently. This method is particularly useful in describing persistences between the different temporal lengths because the decay of ACF is different in various sections. In the previous studies using the method of power spectral density, the attention can not be paid to different temporal lengths. Also, what relationship between the power spectral density and power-law decay of ACF exists has seldom been investigated (Talkner and Weber 2000).

In the last several decades, a method of fluctuation analysis (FA) has been widely used to analyze the persistence of a system (Hurst 1951; Weber and Talkner 2001). FA is essentially the summed squares of the residual found in the window, and is equivalent to an accumulated function of $C(\tau)$ with all possible values of $\tan$ (see Appendix). Later, FA was developed to DFA (detrended fluctuation analysis) (Peng 1994) by considering removal of trend in the window. FA (DFA) produces a slope of power-law for the whole window, indicating an increasing domain averaged residual at a quasi constant rate, but cannot present the correlations between different temporal stages. However, the signal energy shows the strength of persistence at different temporal points. The system persistence should be weaker as the increase of temporal interval. When the temporal interval becomes large enough, the signal energy reaches a saturation state and no signal correlation exists anymore.

In this paper, we investigate the relationship between the signal energy and the decay of ACF, which enables us to use the signal energy to describe the signal correlation in a time series. Because the finite Fourier transform can filter out the signal of random noise, it could be a useful method to measure the variation of ACF for both short-term and longterm persistence, as power-law decay of ACF, does not hold for a longer temporal period. Also, we will compare

To illustrate the signal energy and its association with the surface temperature persistence is the main purpose of this work. The outline of the paper is as follows. In Section 2, we will discuss the basic physics of the Fourier transform of ACF in a discrete form. In Section 3, the ACF will be studied by applying the ECMWF daily data and the leading fluctuations of ACF between any two time points with a non-zero temporal interval are shown. In Section 4, the relationship between the power-law decay of ACF and the signal energy is addressed. Section 5 discusses the results of signal energy presented in a specific portion of the temporal interval. Finally, in Section 6 we conclude with a summary.

\section{Theory of signal energy}

The Fourier transform of a time series $X(t)$ is given by

$F_{x}(\omega)=\int_{-T}^{T} X(t) e^{-j \omega t} d t$

where $\omega$ is the circular frequency, and $T$ is the half length of $X(t)$. The power of the spectral density is defined as,

$S(\omega)=\lim _{T \rightarrow \infty} \frac{\left\langle\left|F_{x}(\omega)\right|^{2}\right\rangle}{2 T}$

where \langle\rangle is the ensemble mean. Substituting Eq. (1) into Eq. (2) gives,

$$
\begin{aligned}
S(\omega) & =\lim _{T \rightarrow \infty} \frac{1}{2 T}\left\langle\int_{-T}^{T} X(t) e^{-j \omega t_{1}} d t_{1} \int_{-T}^{T} X(t) e^{-j \omega t_{2}} d t_{2}\right\rangle \\
& =\lim _{T \rightarrow \infty} \frac{1}{2 T} \int_{-T}^{T} d t_{1} \int_{-T}^{T}\left\langle X\left(t_{1}\right) X\left(t_{2}\right)\right\rangle e^{-j \omega\left(t_{2}-t_{1}\right)} d t_{2} \\
& =\int_{-\infty}^{\infty} C(\tau) e^{-j \omega \tau} d \tau
\end{aligned}
$$

where $C(\tau)=\left\langle X\left(t_{1}\right) X\left(t_{2}\right)\right\rangle=\left\langle X\left(t_{1}\right) X\left(t_{1}+\tau\right)\right\rangle$ is the autocorrelation function, and $\tau=t_{2}-t_{1}$. Equation (3), known as the Wiener-Khinchine relation, is of fundamental importance in analyzing random signals because it provides the link between the time domain of the autocorrelation function and the frequency domain of spectral density. Because of the uniqueness of the Fourier transform, it follows that the autocorrelation function of a random process is an inverse of the transform of spectral density. $S(\omega)$ is called spectrum energy density, which has been demonstrated to obey power-law scaling to frequency, $\omega$ (Talkner and Weber 2000; Weber and Talkner 2001).

Assuming $a>0$ and making the substitution of $\tau^{\prime}=a \tau$, gives

$$
\begin{aligned}
S_{a}(\omega) & =\frac{1}{a} \int_{-\infty}^{\infty} C\left(\tau^{\prime}\right) e^{-j(\omega / a) \tau} d \tau^{\prime} \\
& =\frac{1}{a} S\left(\frac{\omega}{a}\right)
\end{aligned}
$$

Physically, Eq. (4) means that if a signal pulse is compressed in time, the frequency width is expanded, and vice versa, which indicates

$\Delta \tau \Delta \omega \leq \mathrm{const}$

where $\Delta \tau$ is the time duration and $\Delta \omega$ is the width of the transform in frequency space. The result is the Heisenberg uncertainty principle. 
Since $C(\tau)$ is real and $C(-\tau)=C(\tau)$, we only need to consider the real part of Fourier transform in the domain $\tau>0$, and Eq. (3) becomes

$S(\omega)=\int_{0}^{\infty} C(\tau) e^{-j \omega \tau} d \tau$

For a finite data set, the Fourier transform is limited to a finite time domain of $\Delta \tau$, in this domain the basic circular frequency is $\omega_{0}=2 \pi / \Delta \tau$, and we only need consider the frequencies of integer times of $\omega_{0}$, and the Fourier transforms is now

$S\left(n \omega_{0}\right)=\int_{0}^{\Delta \tau} C(\tau) e^{-j n \omega_{0} \tau} d \tau$

where $n=1,2,3, \cdots, N$, and $N=[\Delta \tau]-1 .[\Delta \tau]$ means the integer part of $\Delta \tau$. We don't consider the point of $N=[\Delta \tau]$, since at this point, all cosine functions are equal (or very close) to one. Also, we don't need to consider $n>N$, as the results are repeated in cycle. The summation of all frequencies is

$$
\begin{aligned}
\Delta S & =\frac{1}{\Delta \tau} \sum_{n=1}^{N} S\left(n \omega_{0}\right) \\
& =\frac{1}{\Delta \tau} \int_{0}^{\Delta \tau} C(\tau)\left(e^{-j \omega_{0} \tau}+e^{-j 2 \omega_{0} \tau}+e^{-j 3 \omega_{0} \tau} \cdots e^{-j N \omega_{0} \tau}\right) d \tau
\end{aligned}
$$

$\Delta S$ is called signal energy, which is different from spectrum energy density, $S(\omega)$ in Eq. (4). $S(\omega)$ addresses the spectrum energy at frequency $\omega$ in the whole temporal domain. Whereas $\Delta S$ addresses the spectrum energy in a finite temporal $\Delta \tau$ containing the contributions from all possible frequencies in such domain.

Because $C(\tau)$ is real and we only need consider the real part of $\Delta S$, which is $\operatorname{Re}\left(e^{-j \omega_{0} \tau}+e^{-j 2 \omega_{0} \tau}+\right.$ $\left.e^{-j 3 \omega_{0} \tau} \cdots e^{-j N \omega_{0} \tau}\right) \leq 1$, then $\Delta S \leq \max \{C(\tau)\} \leq 1$, which is the saturation value of $\Delta S$. According to the uncertainty principle of Eq. (5), the wider the time scale, the narrower the width of frequency; thus, the variation of $C(\tau)$ is better resolved. In Eq. (8), when the value of $\Delta \tau$ is larger, more terms of $e^{-j n \omega_{0} \tau} ; n=1,2, \cdots N$ are included.

$\Delta S$ goes up in proportion to the increase of the variation of $C(\tau)$. If $C(\tau)$ is a constant, from Eq. (8) $\Delta S=0$, since the integral for each term of $\cos \left(n \omega_{0} \tau\right)$ is zero. Also, if we assume $C(\tau)$ is in a white noise, $\Upsilon(\tau)$, and $\Delta \tau$ is large enough, we obtain

$$
\begin{aligned}
\Delta S & =\sum_{n=1}^{N} \int_{0}^{\Delta \tau} \Upsilon(\tau) e^{-j n \omega_{0} \tau} d \tau \\
& \approx 0 .
\end{aligned}
$$

This is because the random noise data spreads across the domain between 0 and $n \omega_{0} \Delta \tau=2 n \pi$ and the average of the weight of $\cos \left(n \omega_{0} \tau\right)$ in each term in Eq. (9) is equal to zero (Fante 1988). Therefore, when $C(\tau)$ reaches a random stage, $\Delta S$ will not increase with increasing $\Delta \tau$, as $\Delta S$ is saturated. This is an important point which is used in the following discussions.

\section{Autocorrelation of surface temperature}

Considering a time series of $\psi(t)$, where $t=1,2, \ldots m$ denotes the discretized time, its normalized variability is defined as

$\hat{\psi}(t)=\frac{\psi(t)-\langle\psi(t)\rangle}{\sigma}$,

where $\sigma$ is the standard deviation of $\psi(t),\langle\psi(t)\rangle=\bar{\psi}$ is the time average of the series. The ACF at each spatial point is then given by

$C(\tau)=\langle\hat{\psi}(t) \hat{\psi}(t+\tau)\rangle$.

In the following, we study the ACF and signal energy using the surface screen temperature. The 39-year (1979 to 2017) ECMWF surface screen temperature daily data (Poli et al. 2016), with a global horizontal resolution $2.5 \times 2.5$ degrees are used. The data is de-trended and with annual cycle removed. A reanalysis data is generally much more reliable than the product of the forecast models, since the data assimilation systems is used to reanalysis archived observations.

The ACF provides a measurement of the correlation of the leading fluctuations between any two temporal points with an interval of $\tau$. The statistical average of such correlations is fundamental to examining persistent climate events. If we take the values of ACF as the correlation coefficients, the theory of confidence interval can be applied to the ACF (see Appendix).

In the left column of Fig. 1, the geographical distributions of $C(\tau)$ are shown for several values of $\tau$. (Monahan 2012) has demonstrated the geographical distributions of ACF for surface wind speed. In each panel there are several prominent contour patterns appearing in the land and ocean. We can see that the larger the value of $C(\tau)$, the stronger the correlation in surface temperature between two moments separated by lag $\tau$, which results in a higher probability of climate persistence. The climate persistence and climate variability are different. A signal can have a high variance and either a high or low persistence. The climate variability (generally by standard deviation) emphasizes the deviation to the multi-year climate mean, while climate persistence emphasizes the relationships in climate between neighboring years or within a certain period.

In Fig. 1(a) where $\tau=5$ days, the $C(\tau)$ represents the statistical average of the correlation between every two 

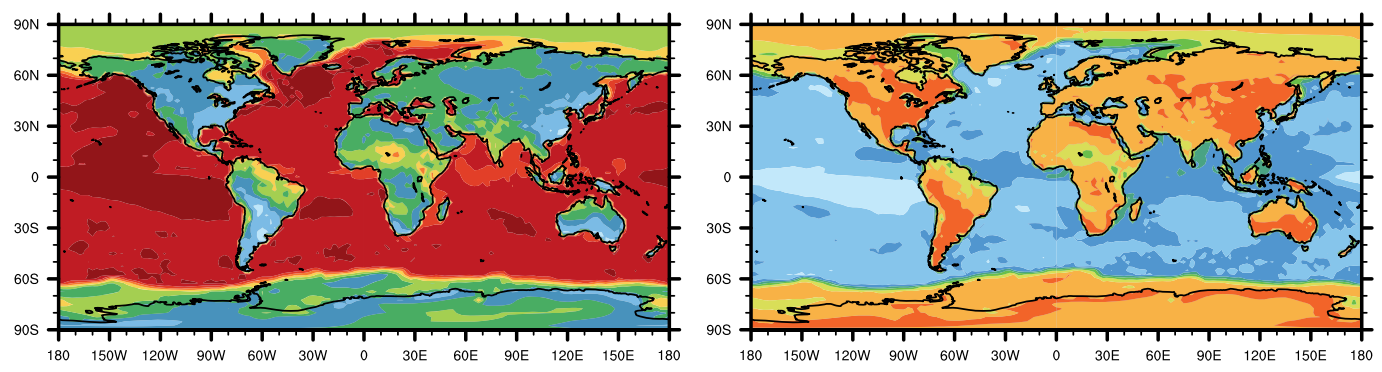

(c) $\tau=14$ day

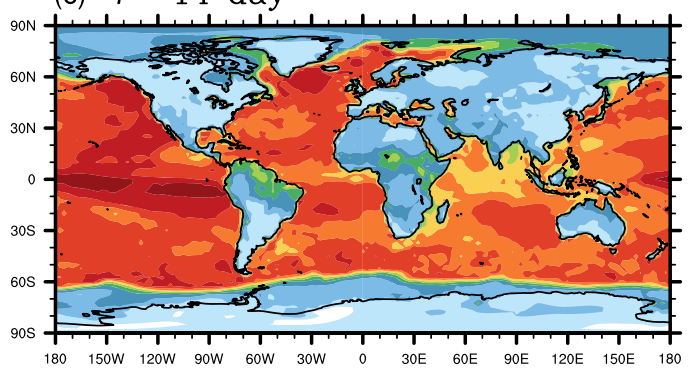

(c)

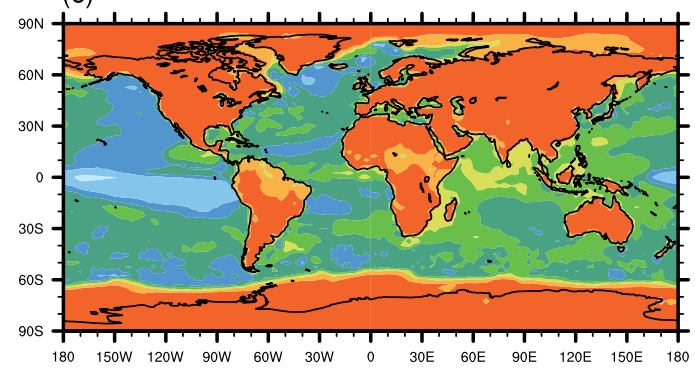

(e) $\tau=30$ day

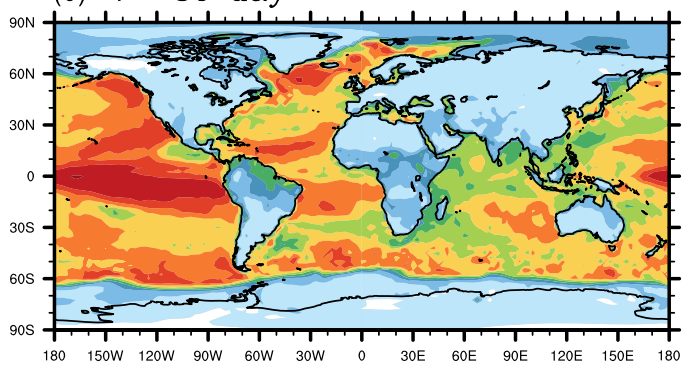

(f)

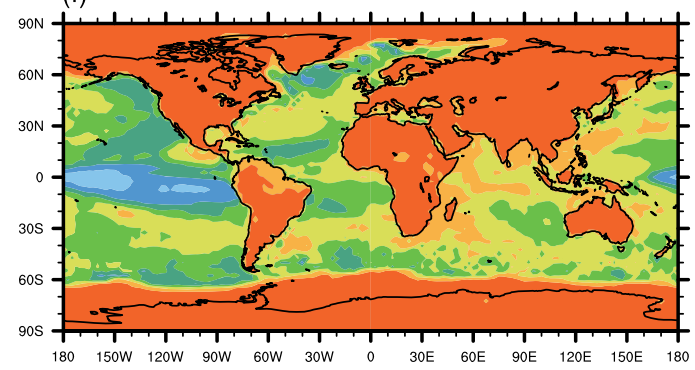

(g) $\tau=90$ day

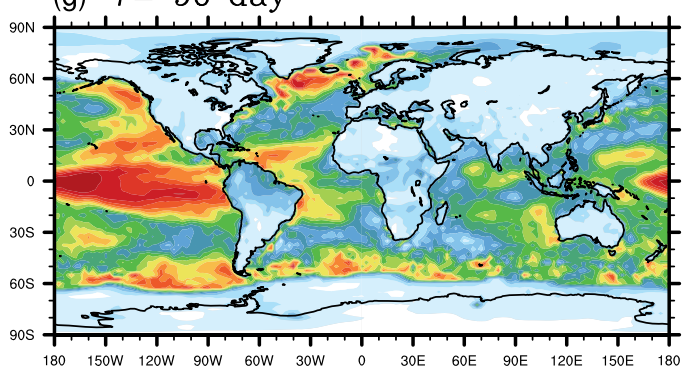

(h)
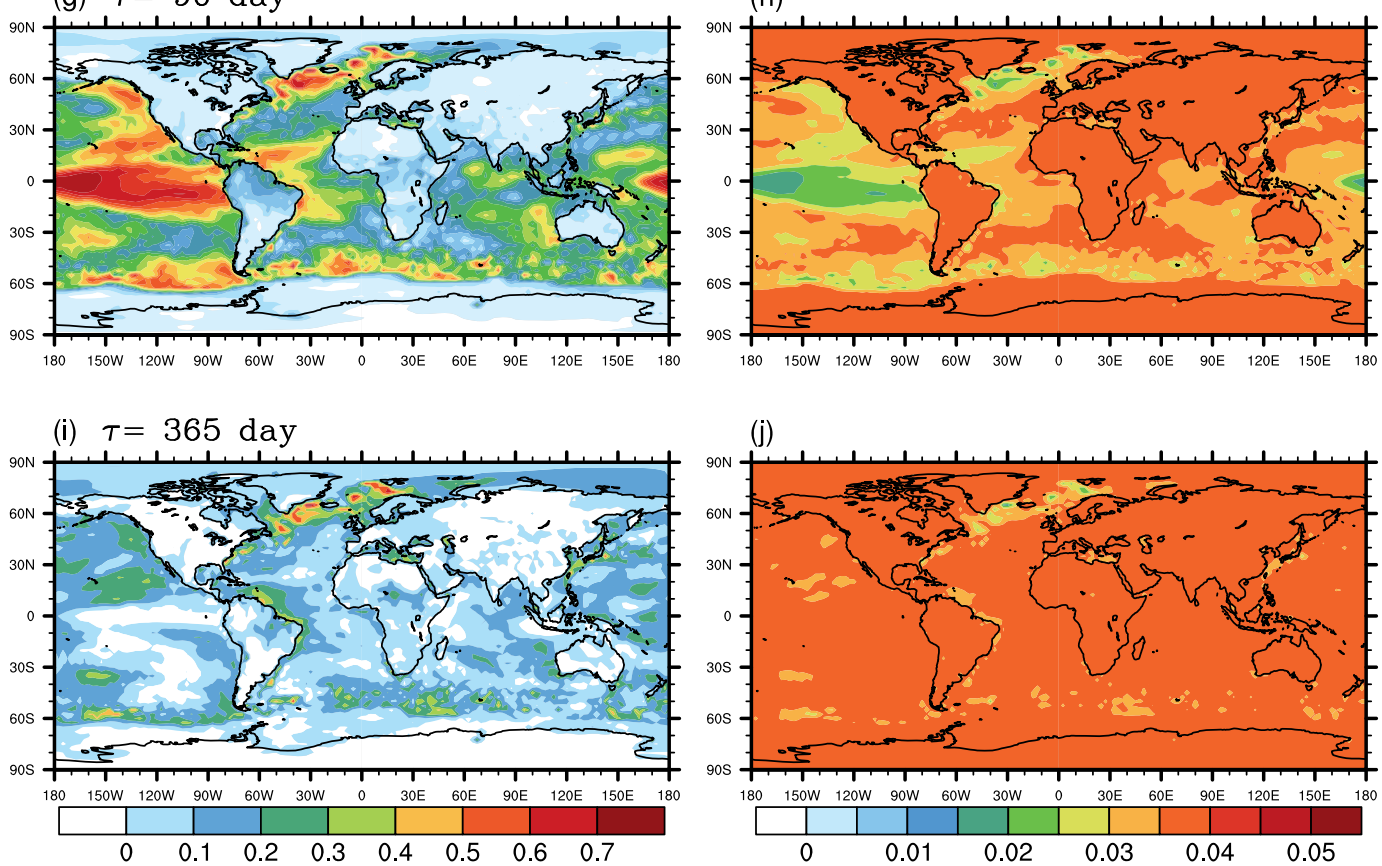

Fig. 1 The left column shows the distributions of $C(\tau)$ for different values of lag, $\tau$. The right column shows the corresponding results of confidence interval. Based on (1979 to 2017) ECMWF daily data 
moments separated by 5 days, which shows the climate persistence in such period. The distribution of $C(\tau)$ displays persistence probability of the surface temperature in every 5 days. In the North American continent, the west part has relatively larger $C(\tau)$ compared to the east part, as the persistence of surface temperature is higher in the western part of America. The tropical region in Africa also has a relatively large value of $C(\tau)$, as the land surface temperature in the tropics is more stable. In contrast with the land, the values of $C(\tau)$ over the ocean are very close to one. Therefore, the change in ocean daily temperature in a 5 -day period is small.

The confidence intervals of ACF are seen in the right column. These depends on three factors: the confidence level set, the value of the correlation coefficient as given by the ACF value, and the length of data. The confidence level here is set to 0.95 , and the length of data set is 14244. The confidence interval indicates a valid range for the confidence level. The larger the confidence interval, the less the possibility of reaching the required confidence level. In our case, the results are for a 95\% probability to appear in the range of [mean - $0.5 x$ (confidence interval), mean + $0.5 x$ (confidence interval)], the confident interval is equal to upper limit minus lower limit (see Appendix)

Generally, the confidence interval should be less than 0.1 to obtain a reliable result. In $\tau=5$, the confidence interval is less than 0.04 over the land and less than 0.02 over the ocean, indicating the very reliable results of the autocorrelation calculations.

The second row shows the results of $C(\tau=14)$ that relates confidence intervals. The values of $C(\tau)$ decreases in both land and ocean compared to the results of $C(\tau=5)$, as the probability of persistence decreases with the increase of time intervals. Also, the decreasing rate of $C(\tau)$ is not homogeneous, as the patterns of $C(\tau=14)$ are not the same as $C(\tau=5)$. In northern America, the difference of $C(\tau)$ in the west and east parts becomes smaller compared with $C(\tau=5)$. The corresponding confidence interval increases and the reliability of the correlation decrease with the increase of the temporal intervals.

The third and fourth rows present the results of $C(\tau=$ $30)$ and $C(\tau=90)$. The values of $C(\tau)$ become less than 0.1 in the North American and Eurasian

continents as the temporal correlations become very weak. This implies that a long time weather forecast is very challenging because the climate persistence almost does not exist as the fluctuations increases. Over the ocean, the correlation coefficient decreases with $\tau$ as well. The most characteristic feature is that the values of $C(\tau)$ do remain relatively large in the El Nino region, as the surface temperature there has a small variation in a time length less than 90 days, because El Nino is a longer time scale event. The confidence interval over the ocean increases compared to that $C(\tau=14)$, but it is almost unchanged over the land because of the subtle difference in $C(\tau)$ there.

$C(\tau=365)$ shown in the bottom row represents the persistence in surface temperature in every two moments with a one-year gap. The correlation between these two moments becomes very weak even in the ocean. The remarkable difference between $C(\tau=365)$ and $C(\tau=$ 90) occurs in the El Nino region, where the correlation coefficient dramatically reduces to nearly zero. Therefore, from $\tau=90$ to 365 days, the decay of $C(\tau)$ is more substantial over the El Nino region compared to other oceanic areas. Also, the confidence interval over the ocean increases, and now the values over land and ocean become very close.

\section{ACF power-law decay and signal energy}

The last section shows that the values of ACF decay with increasing $\tau$. What kind of governing rule for the decay of ACF is an interesting problem. In atmospheric science, it is generally claimed that the autocorrelation follows the power-law decay, which is called a long-range correlation. If ACF follows a power-law decay then

$C(\lambda \tau) \sim \tau^{-\alpha}$,

which implies that the scaling rule exists as

$C(\lambda \tau)=\lambda^{-\alpha} C(\tau)$.

From Eq. (12), the smaller the $\alpha$, the larger the degree of persistence. When $\alpha \rightarrow 0$, there is no change in $C(\lambda \tau)$ and $\alpha$ also cannot be negative.

However, so far there lacks a theoretical proof for Eq. (13). In the atmosphere, the power-law scaling of ACF has been tested before (Koscielny-Bunde et al. 1996; Talkner and Weber 2000; Eichner et al. 2003). Here we are interested in examining the longer time scales over a broad geographical domain, to find the extent of the power-law decay for ACF and its relationship with signal energy.

In Table 1, we have chosen 8 locations over land, and 8 locations over the ocean. Over the land, there are two locations in each of North America, South America, Africa, and Eurasian Continent. Over the ocean, there are four locations in the northeastern and central Pacific, Nino $1+2$ and Nino 3.4, and two locations in each of the Atlantic, and Indian Ocean. For Nino $1+2(90 \mathrm{~W}-80 \mathrm{~W}, 0-10 \mathrm{~S})$, we choose the point of $(85 \mathrm{~W}, 5 \mathrm{~S})$ to represent it, and for Nino $3.4(170 \mathrm{~W}-120 \mathrm{~W}, 5 \mathrm{~N}-5 \mathrm{~S})$ we choose the point of (140 $\mathrm{W}, 0 \mathrm{E})$.

Figure 2(a) shows the results of ACF over the land, in the natural $\log$ arithm coordinate of $\log C(\tau)$ and $\log \tau$. The maximum lag of $\tau$ is 360 days, and usually the maximum $\tau$ should not exceed one-quarter of the length of the time 
Table 1 The locations of the chosen points

\begin{tabular}{lll}
\hline Color of line & Land & Ocean \\
\hline Red & Western Canada $(52.5 \mathrm{~N}, 127.5 \mathrm{~W})$ & Northwestern Pacific $(52.5 \mathrm{~N}, 175 \mathrm{E})$ \\
Orange & Southeastern America $(37.5 \mathrm{~N}, 117.5 \mathrm{~W})$ & Central Pacific $(37.5 \mathrm{~N}, 152.5 \mathrm{~W})$ \\
Brown & Central Latin America $(15 \mathrm{~S}, 45 \mathrm{~W})$ & Nino $1+2(5 \mathrm{~S}, 85 \mathrm{~W})$ \\
Blue & South Latin America $(35 \mathrm{~S}, 60 \mathrm{~W})$ & Nino $3.4(0,140 \mathrm{~W})$ \\
Green & Northern Africa $(17.5 \mathrm{~N}, 0)$ & Northern Atlantic $(40 \mathrm{~N}, 30 \mathrm{~W})$ \\
Yellow & Central Africa $(0,27.5 \mathrm{E})$ & Central Atlantic $(15 \mathrm{~N}, 30 \mathrm{~W})$ \\
Purple & Central Europe $(47.5 \mathrm{~N}, 32.5 \mathrm{E})$ & Northern Indian Ocean $(2.5 \mathrm{~S}, 70 \mathrm{E})$ \\
Gray & Central China $(35 \mathrm{~N}, 112.5 \mathrm{E})$ & Southern Indian Ocean $(17.5 \mathrm{~S}, 72.5 \mathrm{E})$ \\
\hline
\end{tabular}

series in any ACF calculations. For $\log \tau<4 \quad(\tau<60$ days ), a decay of $C(\tau)$ is clearly displayed; however, Eq. (12) is not satisfied, since the power-law requires a linear relationship between the $\log C(\tau)$ and $\log \tau$ coordinates. If this relationship is not linear, then the decay rate of ACF is different in different regions. We find that the gradients of the curves steepen with $\tau$, indicating a more substantial decay at a larger $\tau$. For large values of $\log \tau>4$, strong fluctuations occur and the results of $C(\tau)$ become random noisy; thus, no correlation exists anymore.

\section{Land}

(a)

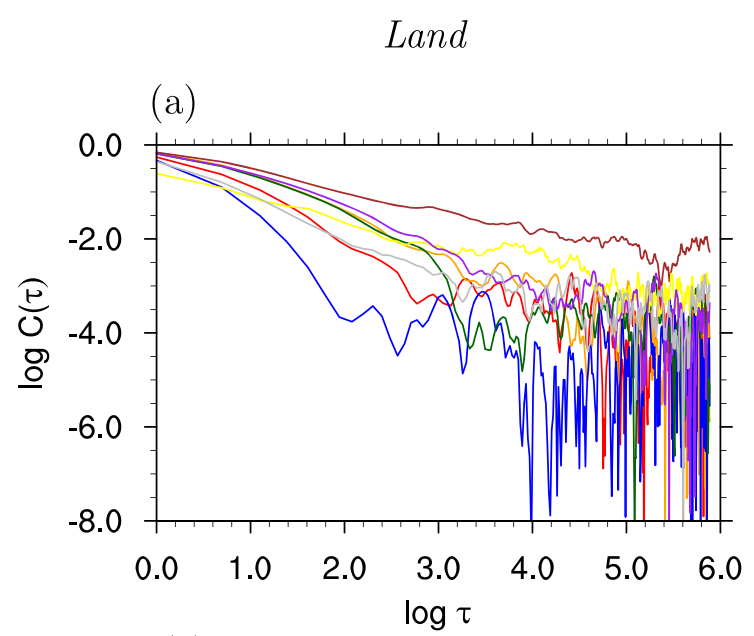

(c)

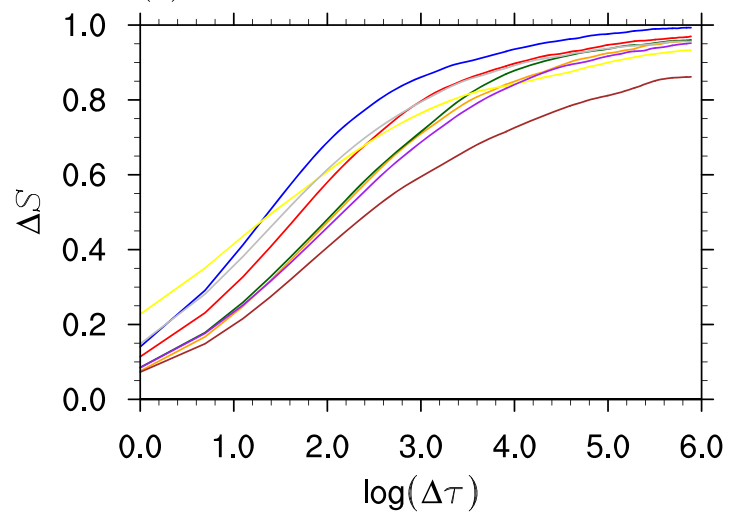

In Fig. 2(c), the corresponding results of $\Delta S$ are shown. In the region of $\Delta \tau<60$ days, $\Delta S$ increases dramatically. As mentioned above, the large values of $\Delta S$ are caused by the large variations of $C(\tau)$ in the each region. For example, the blue line of $C(\tau)$ has the largest decay rate, the corresponding curve of $\Delta S$ shows the largest increase with the increase of $\tau$. The curves of $\Delta S$ are not parallel to each other in the regions of relatively small $\Delta \tau$, as the changes of signal energy in different regions are very different. When $\Delta \tau>60$ days, the values of $\Delta S$ increase slowly.

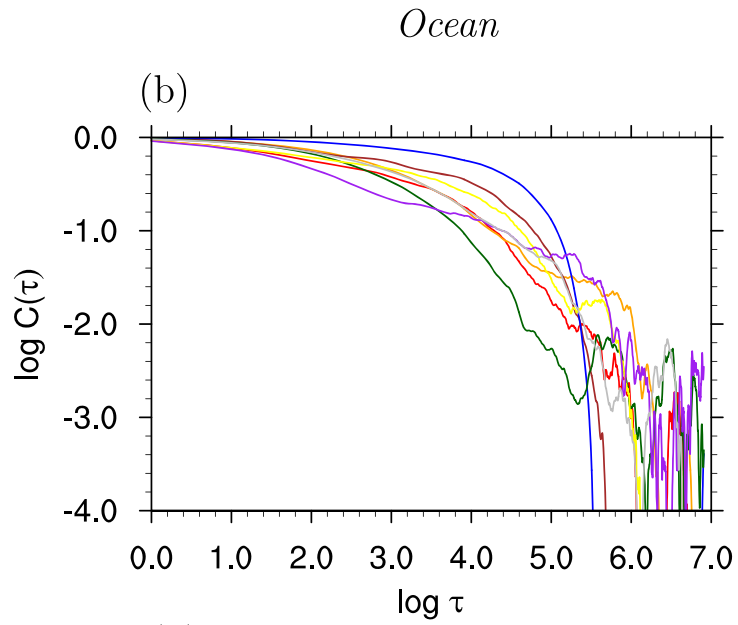

(d)

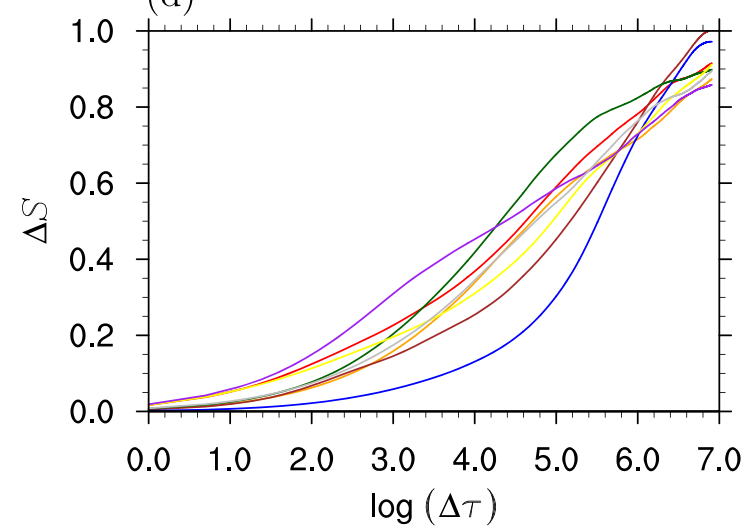

Fig. 2 Top panels are the results of $C(\tau)$ for surface temperature based on 16 locations from Table 1; Bottom panels are the results of signal energy, $\Delta S$. The left and right columns are the results of land and ocean, respectively 
Especially when $\Delta \tau>180$ days, the changes of curves become minimal, and all the curves are close to parallel to each other. Under this condition, no more information is provided. As mentioned above, when $C(\tau)$ reaches a random noisy region, it has no contribution to the signal energy, so that the change in $\Delta S$ becomes very small and $\Delta S$ is saturated.

Figure 2(b) shows the results of ACF over the ocean. Different from the land, the decay rate of $C(\tau)$ becomes much slower, as the correlation of surface temperature over the ocean is stronger compared to the land. The daily persistences are larger compared to the land since the smaller decay of $C(\tau)$ leads to a stronger similarity in climate states. Though the decay of $C(\tau)$ is shown for all curves, the power-law relation of Eq. (12) does not exist either, as the different regions present different decay rate. When $\log \tau$ is close to 6 ( $\tau \approx 400$ days), the extreme fluctuations happen, and the curves become random noise.

Figure 2(d) shows the corresponding results of $\Delta S$. Different from the land, the values of $\Delta S$ increase relatively slow. The smaller $\Delta S$ is caused by the lower variation of $C(\tau)$ over the ocean. For example, the blue line of $C(\tau)$ has the slowest decay rate, and the corresponding curve of $\Delta S$ shows the smallest increase with $\tau$. Also, the changes in signal energy in different regions are very different, which evaluate the differences in variation of surface temperature. When $\Delta \tau>400$ days, the values of $\Delta S$ increase slowly, as $\Delta S$ is saturated because most of the curves of $C(\tau)$ are in the noisy state. However, the results $\Delta S$ of the blue and brown curves continuously increase till $\Delta \tau$ reaches about 900 days, because the corresponding curves of $C(\tau)$ approach the random noise state relatively slow compared to other curves.

In order show that the results of Fig. 2 are universal, in the Appendix we have applied the signal energy to the time series of ECMWF daily data of geopotential height at 500 $\mathrm{hPa}$. Also the comparisons to ACF and FA are presented.

In Fig. 3, we further study the detailed geographical distributions of the critical exponents, similar to the works of Monettia et al. (2003) for the scaling of the fluctuation function as applied to the sea surface temperature data. From Fig. 2, the critical exponents of ACF are not linear (as straight line); thus, the geographical distributions of $\alpha$ are different for different values of $\tau$. A linear regression method is used to obtain the value of $\alpha$ in the linear relation of $\log C(\tau)=-\alpha \log \tau$ for different lengths of $\tau$.

Figure 3(a) shows the result of $\Delta \tau=5$ days, the distribution of $\alpha$ is small and very homogeneous over the ocean. This is caused by the maximum persistence there as shown in Fig. 1. It is interesting to find that $\alpha$ is close to zero in the El Nino region. In the short period of 5 days, the correlation of surface temperature in the El Nino region is very strong and the value of ACF is close to one (see Fig. 1).
The critical exponents of ACF become much larger over the land compared to the ocean, as the climate persistence is weaker. It is interesting to find that the value of $\alpha$ is relatively large in the southeast part of North America, but relatively small in the west part. The day to day variation in western part of North America is smaller than that in the southeast part, which is also consistent with Fig. 1. ACF shows the correlation between any two moments, while the decay rate of $\alpha$ shows the averaged variation during the period between these two moments. They are different but associated, usually a smaller autocorrelation in a period corresponding to a larger decay rate. Over South America, the Eurasian continent, and Australia, the large contrasts in $\alpha$ happen as well.

Figure 3(b) shows the corresponding result of $\Delta S$. The distribution of $\Delta S$ is very similar to that of $\alpha$, with a correlation coefficient of 0.97457 . All the main features shown in the distribution of $\alpha$ appear in the distribution of $\Delta S$. The larger the $\Delta S$, the larger signal energy, as the ACF has a larger decay rate in this period with less chance for persistence. For example, the large value of $\Delta S$ in the southeastern North America indicates a weak persistence of ACF, and at the same time, the temperature correlations in a 5-day period are small in this region compared to the western North America.

When $\Delta \tau=14$ days (second row), the values of $\alpha$ rise over the western North America, as a larger decay of ACF occurs, which means a smaller persistence in this period. Compared to the results of $\Delta \tau=5$ days, some regions, such as Greenland and Eurasian continent, show obvious changes in $\alpha$; other regions, such as North Latin America and central Africa, show minimal changes. The correlations of surface temperature are very different in different regions. In an area with a larger change in $\alpha$, the variations of surface temperature are larger. Overall the values of $\alpha$ increases for $\Delta \tau=14$ days compared to $\Delta \tau=5$ days. The decrease in the persistence of surface temperature results in less potential for surface temperature predictions.

The correlation coefficient between $\Delta S$ and $\alpha$ is 0.94964 , as the two patterns are still very similar at $\Delta \tau=14$ days. Similar to the distribution of $\alpha$, the signal energy in the western North America increases, as the possibility of persistent surface temperature becomes very small in a 14-day length scale. are included. Also the values of $\Delta S$ increase over all other continents. The values of $\Delta S$ are relatively small near central Africa, South America, and South India, as the day to day persistence in the tropics is stronger compared to the extra-tropics. Over the ocean, relatively large values of $\Delta S$ appear in the Indian Ocean, signal energy is accumulated there for a 14-day period.

When $\Delta \tau=30$ days (third row), the values of $\alpha$ over the northwestern part of North America become even larger compared to that of $\Delta \tau=14$ days. However, the values of 


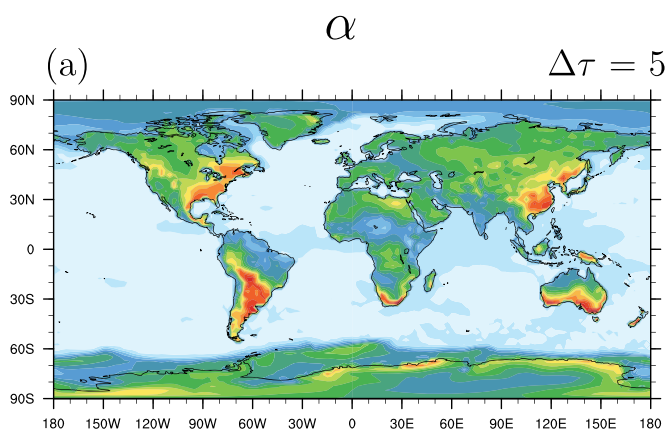

$\Delta S$

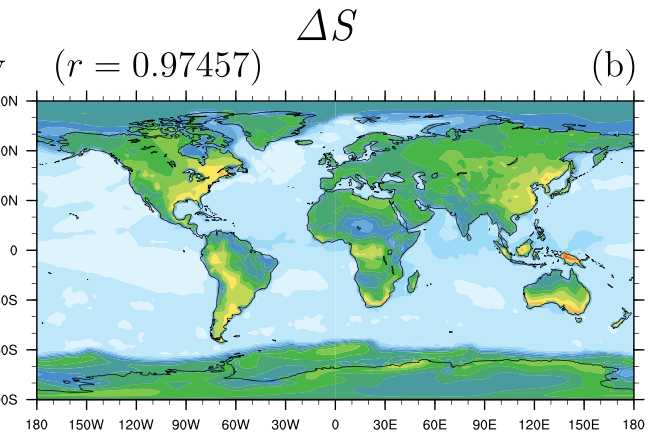

(c)

$\Delta \tau=14$ day $\quad(r=0.94962)$
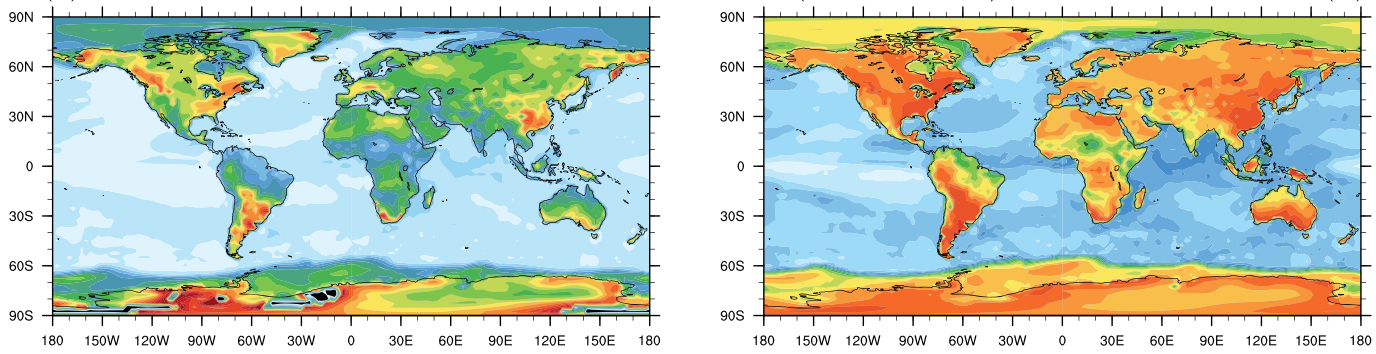

(e)

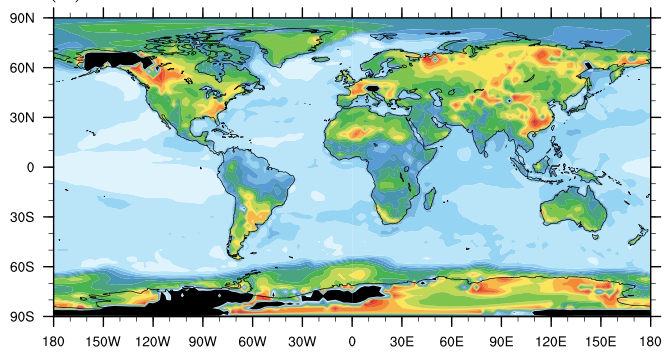

$\Delta \tau=30$ day $\quad(r=0.93205)$

(f)

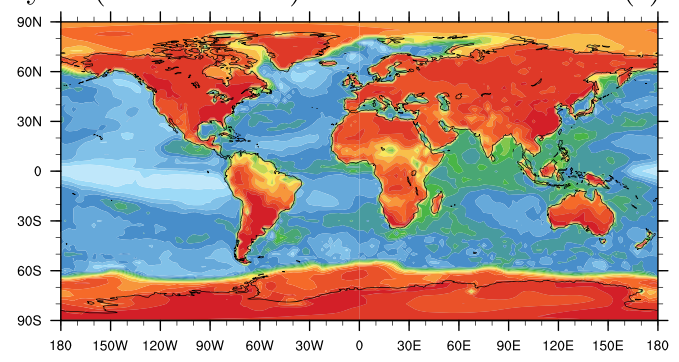

(g)

$\Delta \tau=90$ day $\quad(r=0.65153)$

(h)
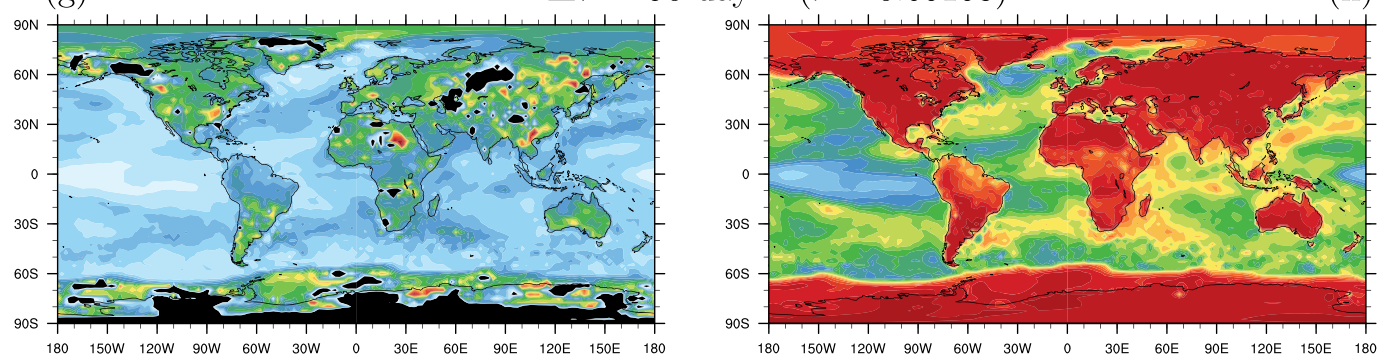

(i)

$\Delta \tau=365$ day $\quad(r=0.37499)$

(j)
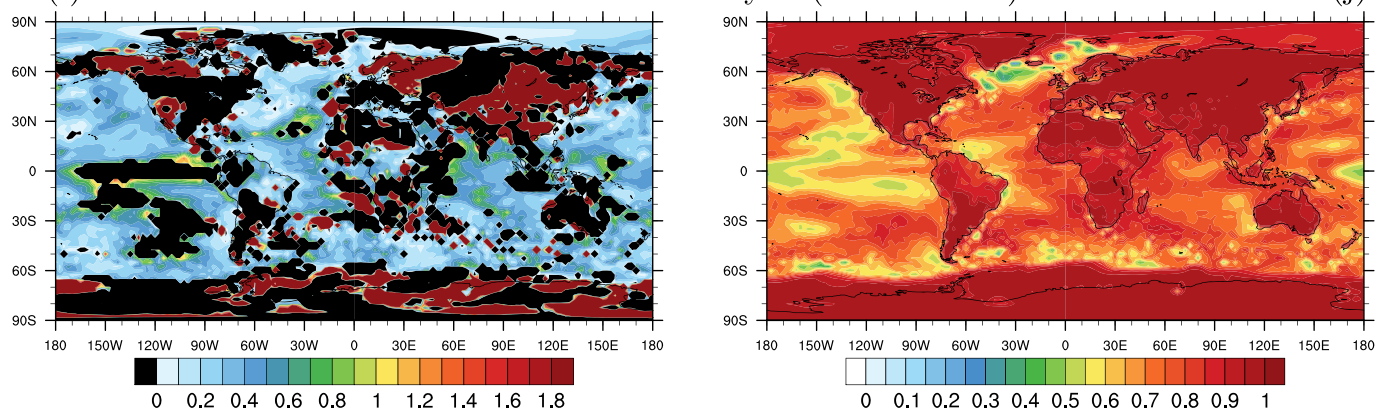

Fig. 3 The distributions of the critical exponent $\alpha$ with different lengths of $\Delta \tau$ (left column), and the corresponding results of $\Delta S$ (right column). $r$ is the correlation coefficient between $\alpha$ and $\Delta S$ 
$\alpha$ over the lower eastern North America decrease, indicating an increase of correlation in surface temperature at a larger temporal interval, which is physically unrealistic. From Fig. 2, the decay of ACF breaks down for a larger value of $\tau$, as the curves show the random state when $\tau>20$ days, which explains in such nonphysical results. Over the land, there are several places marked as black, in these regions the values of $\alpha$ are negative, which means the decay of ACF becomes invalid, and the results of ACF turns to a noisy state. However, there is no black mark over the ocean at this temporal length period. As shown in Fig. 2, the curves of $\alpha$ over the ocean are not in the random noisy state when $\Delta \tau=30$ days.

The correlation coefficient between $\Delta S$ and $\alpha$ at $\Delta \tau=$ 30 is 0.93205 for the valid regions of $\alpha$, mostly dominated by the similarity over the ocean. Though there are invalid places in the distribution of $\alpha$, the nonphysical phenomenon does not appear in the distribution of $\Delta S$, as the values of $\Delta S$ always increase with $\Delta \tau$ (Fig. 2). The longer the period, the larger the signal energy and the less chance in persistence of the surface temperature.

When $\Delta \tau=90$ days (fourth row), the black marks appear in many places over the land. Based on Fig. 2, now most curves of $\alpha$ are in random noise states. Therefore, the rule of ACF decay does not apply to the land. Over the storm track regions in the Pacific and Atlantic, the values of $\alpha$ are obviously larger than those of $\Delta \tau=30$ days, as the persistence of surface temperature is weaker there. Also, the relatively larger values of $\alpha$ appear in the subtropical jet stream region over the southern hemisphere. In the tropical western Pacific, the values of $\alpha$ are small over the Nino 3.4 region, as the timescale of ENSO is much longer than 90 days; thus, the large variation does not show at this timescale. The correlation coefficient between $\Delta S$ and $\alpha$ at $\Delta \tau=90$ is 0.65153 in the valid regions of $\alpha$. The values of $\Delta S$ are close to one over all the continents, as the signal energy is saturated and there is no autocorrelation for surface temperature during such an extended period. Over the ocean, the results of $\Delta S$ are still far from saturation. Relatively large values appear in the storm track regions. Also, the relatively small values appear in the Nino 3.4 region, similar to the distribution of $\alpha$.

The bottom row presents the result of $\Delta \tau=365$ days. Even over the ocean, Fig. 2 shows that the strong fluctuations happen at $\tau>250(\log \tau>5.5)$ days. Therefore, the critical index, $\alpha$, has lost the physical meaning. In many high latitude regions, the nonphysically negative values of $\alpha$ are marked in black. The results in most regions are unreasonable, because the values of $\alpha$ decrease compared to $\Delta \tau=30$ and 90 days. At this time length, $\Delta S$ is almost completely saturated over the land. In contrast with the noisy distribution of $\alpha$ in the ocean, the distribution of $\Delta S$ exhibits clear patterns with clear physical meaning. It is interesting to find that the smallest values of $\Delta S$ appear in the Labrador sea and near South Greenland. The Labrador Sea is a major source of the North Atlantic Deep Water, a cold water mass that flows at great depth along the western edge of the North Atlantic, which causes a relatively stable sea surface temperature near the coast of Labrador and Newfoundland; thus, the chance of surface temperature persistence is relatively large. A similar finding in relatively stable surface temperature was also found and analyzed by Gerhard (2000). The value of signal energy in Nino 3.4 is larger than that of Nino $1+2$, as the interannual variability is more significant in the region of Nino 3.4 (Chylek et al. 2018).

In summary, over the land the decay rule of ACF can describe a very short time scale of surface temperature persistence, probably the upper limit is about to 14 days, which helps to understand the upper limit of skilful weather forecasts is about 14 days (Lorenz 1963). Over the ocean, the ACF decay can persist for about 90 days because the temperature variation is much weaker over the ocean compared to the land. Generally, the ACF scaling is not true, otherwise, the patterns of the distribution of $\alpha$ should be the same regardless of the length of $\Delta \tau$. The distributions of $\Delta S$ provide an effective method to investigate the correlation in different lengths of time. For a short period, the distribution of $\Delta S$ is very similar to that of $\alpha$, with a correlation coefficient over 0.97 . Both methods provide a measurement of the change in correlations between different temporal moments. As $\Delta \tau$ increases, the nonphysical results occur for the decay of ACF. However, $\Delta S$ always presents very stable results, because the method of signal energy automatically filters out the noisy information and provides a saturated upper limit.

In Fig. 4, we further consider the regional results of the northern hemisphere above $20^{\circ} \mathrm{N}$. The distributions of $\Delta S$ are shown for winter of December, January and February (DJF) as the boreal winter and June, July and August (JJA) as the boreal summer. At $\Delta \tau=5$ days, the distributions of $\Delta S$ are very different between the boreal winter and summer. In the boreal winter of North America, the relatively large values of $\Delta S$ appear in the southern part; thus, the surface temperature has less chance for persistence. The result of the boreal summer is the opposite, as the temperature variation in a 5-day period is more substantial in the northern part. Therefore, in northern America, the surface temperature in a 5-day period is more stable in the winter season compared to the summer season; in southern America, the result is the opposite. In the eastern part of Eurasian Continent, larger values of $\Delta S$ appear in the summer season, but the western part of the Eurasian Continent is less seasonally dependent.

When $\Delta \tau=14$ days, the values of $\Delta S$ increase over the land and the distributions $\Delta S$ become more homogeneous. 
Boreal Winter

(a)

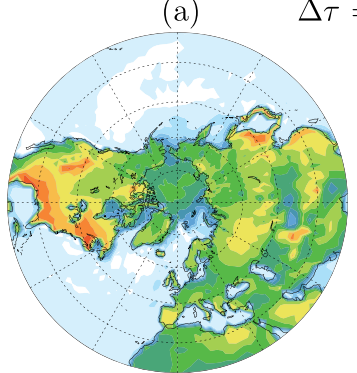

(c)

$\Delta \tau=14$ day

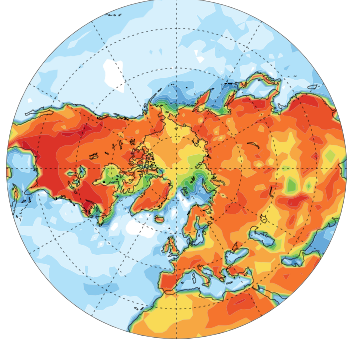

(e) $\Delta \tau=30$ day

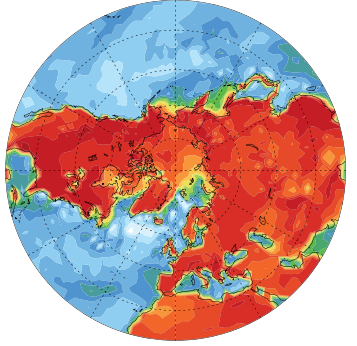

(g) $\quad \Delta \tau=90$ day

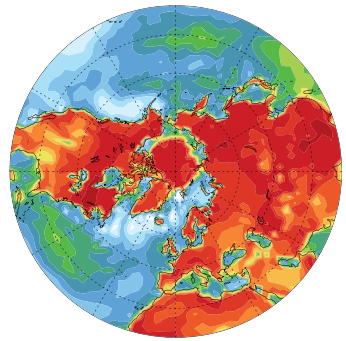

(h)
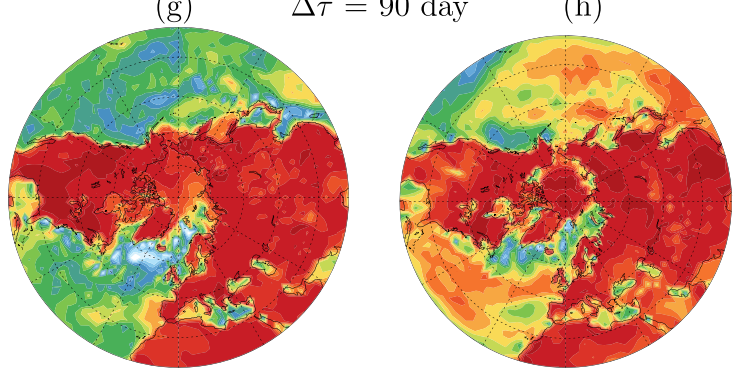

(i)

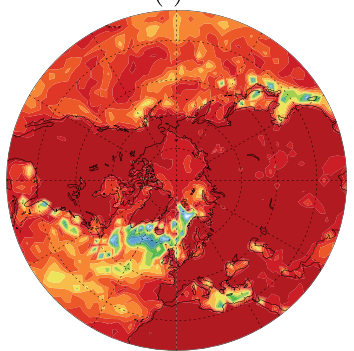

(j)

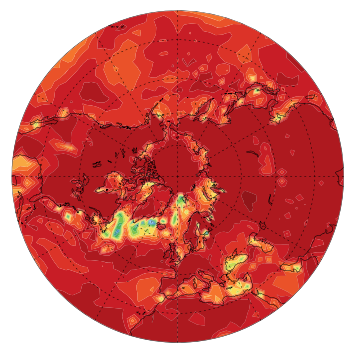

Fig. 4 The left and right panels are the $\mathrm{NH}$ distributions above $20^{\circ} \mathrm{N}$ of $\Delta S$ for the boreal winter and summer with different values of $\Delta \tau$

However, the differences in $\Delta S$ between the winter and summer seasons are still distinguishable over the northern America and Eurasian Continent.
When $\Delta \tau=30$ days, the result of $\Delta S$ over the land is very close to the saturation value of one. Over the ocean, in both regions of Kuroshio and Gulf stream, the values of $\Delta S$ are relatively larger in the summer season compared to the winter season, over these regions the surface temperature has more persistent relation in the winter season compared to the summer season.

When $\Delta \tau=90$ days, a characteristic pattern exhibits in the storm track region over the northern Atlantic with relatively large $\Delta S$, as the surface temperature has strong variation and weak temporal correlation in this period.

When $\Delta \tau=365$ days, the values of $\Delta S$ in both the land and ocean are close to the saturation value, so there is no values in examining the change of correlation in surface temperature over such an extended period. A relatively small $\Delta S$ does appear in the polar region of North Atlantic. The persistence of the sea surface temperature indicates a maximum persistent correlation, which is consistent with other studies (de Coëtlogon and Frankignoul 2003).

\section{Signal energy in a portion of temporal interval}

The Fourier transform is sensitive to the range of the integral it uses. In Fig. 2, the signal energy $\Delta S$ is an accumulated results as $\Delta \tau$ starts from $\tau=0$. In this section, we look at time range that do not just start from $\tau=0$ as done previously. Here we consider $\Delta S$ for a certain interval of $[\Delta \tau]=\tau_{2}-\tau_{1}$, which reveals the signal energy in this period and we denote the signal energy in this period as $[\Delta S]$.

$[\Delta S]=\frac{1}{[\Delta \tau]} \sum_{n=1}^{N}\left[S\left(n \omega_{0}\right)\right]$

where

$\left[S\left(n \omega_{0}\right)\right]=\int_{\tau_{1}}^{\tau_{2}} C(\tau) \cos \left(n \omega_{0} \tau\right) d \tau$

From Fig. 2, $\Delta S$ increases dramatically when $\Delta \tau$ is close to zero, and $\Delta S$ eventually approaches the saturation at a large $\Delta \tau$. Therefore, if $\Delta S$ has reached the saturation state at $\tau_{1},[\Delta S]$ becomes very close to zero for any $\tau_{2}>\tau_{1}$.

Figure 5 shows the results of signal energy in 6 pieces of time. In the period of $[\Delta \tau]$ from 60 to 90 days, the value of $[\Delta S]$ over the land is close to zero, since $\Delta S$ is close to a saturation state at $\Delta \tau=60$ days (see Fig. 2). The relatively large values of $[\Delta S]$ only appear over the ocean, such as in the southern hemispheric jet stream region, indicating the change of persistence in this period. 
(a)

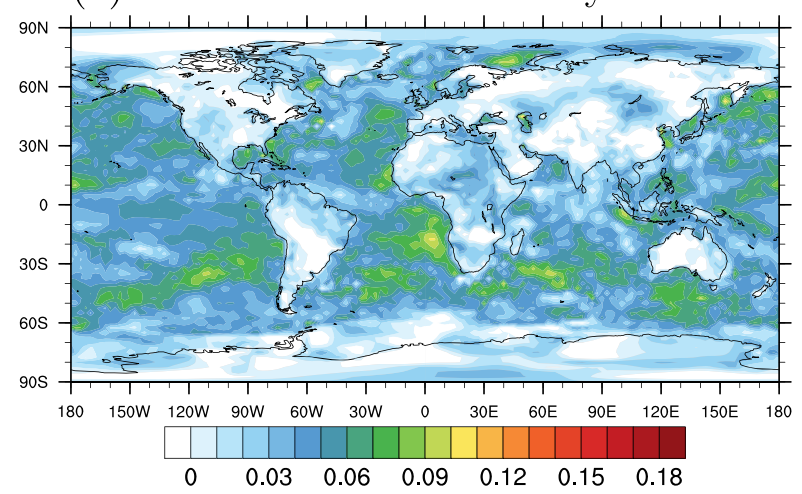

(c)

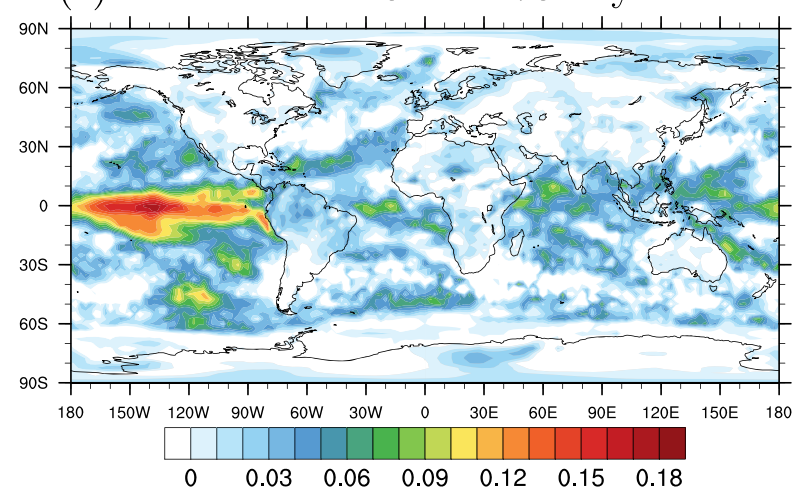

(e)

$\Delta \tau=365$ to 455 day

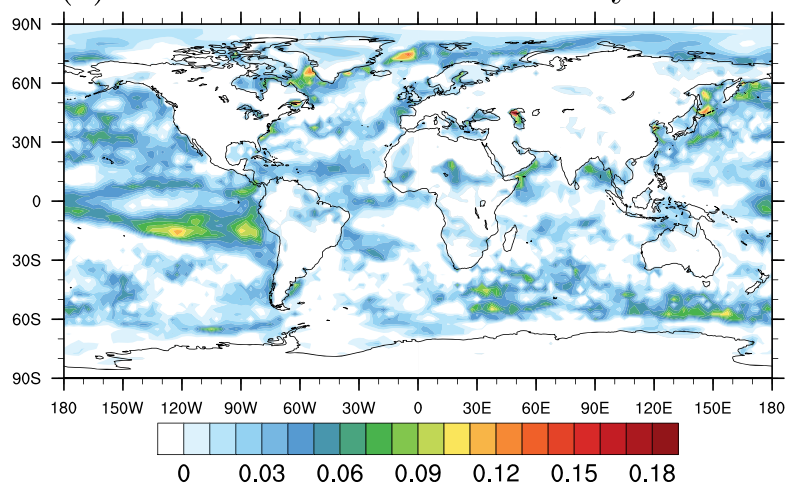

(g)

$\Delta \tau=546$ to 637 day

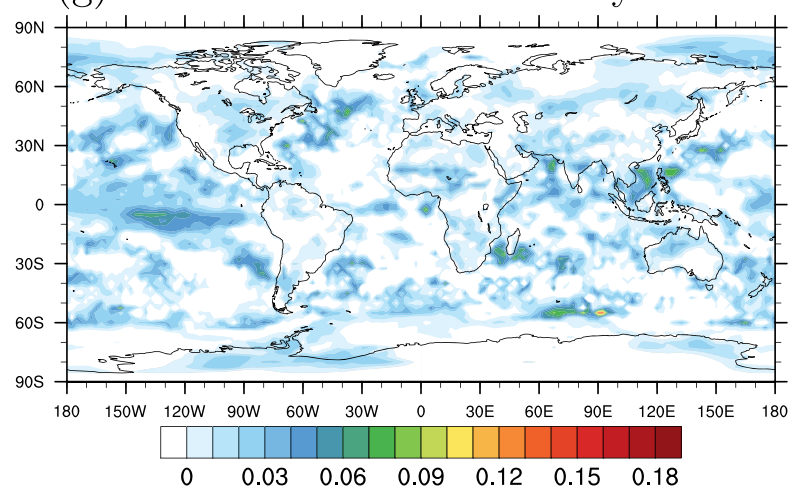

(b) $\Delta \tau=90$ to 182 day

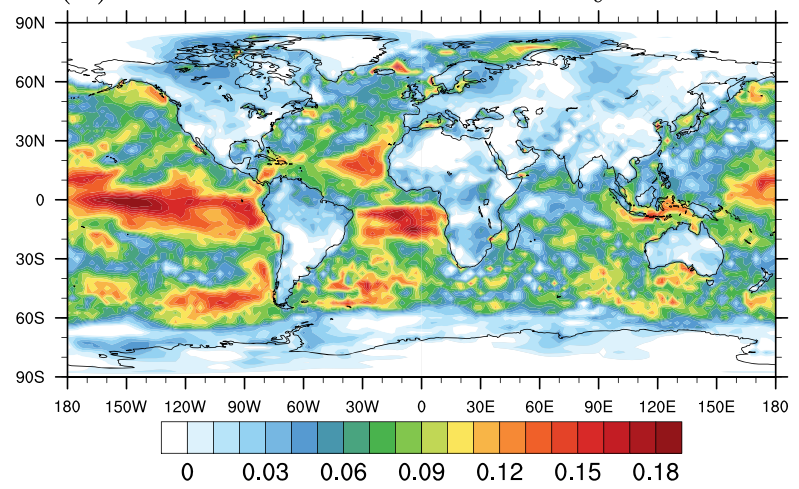

(d)

$\Delta \tau=273$ to 365 day

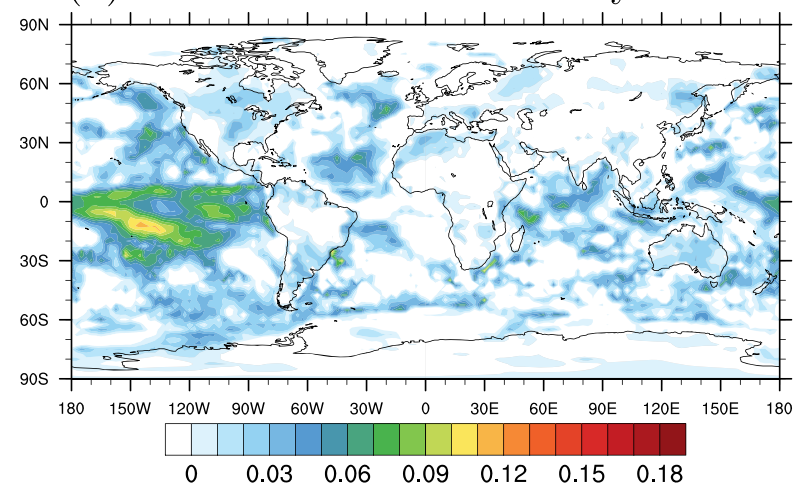

$\Delta \tau=455$ to 546 day

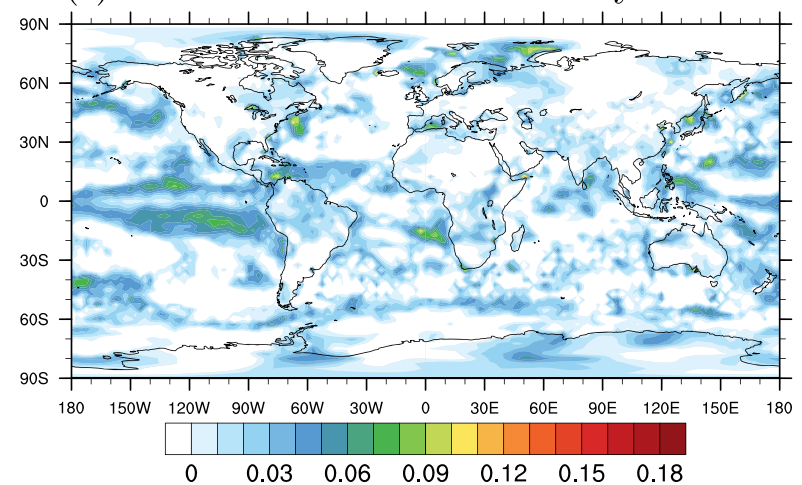

(h)

$\Delta \tau=637$ to 730 day

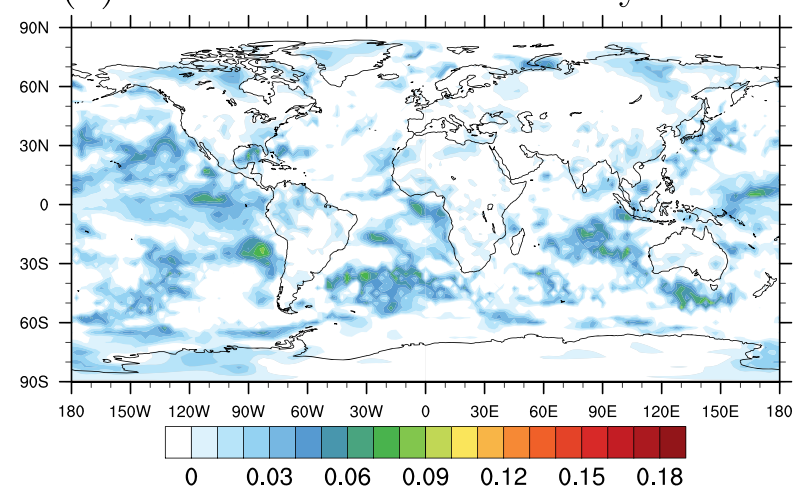

Fig. 5 The distributions of $[\Delta S]$ for different interval of $\Delta \tau$ 
When $[\Delta \tau]$ is between 90 and 182 days, it is interesting to find that $[\Delta S]$ increases dramatically in the El Nino region. Also, there are large values $[\Delta S]$ over the tropical Atlantic. Based on Fig. 2, though $C(\tau)$ does not obey the power-law, but the decay always occurs before it reaches a random state. The larger the decay of $C(\tau)$, the larger the $\Delta S$. Therefore, in these regions the correlations of surface temperature decrease dramatically in the period between 90 and 182 days.

For $[\Delta \tau]$ between 182 and 273 days, the values of $[\Delta S]$ in the El Nino region decreases especially in the area of Nino $1+2$. As it is mentioned above, $\Delta S$ is equal to zero when $C(\tau)$ is a constant or in a random state. Therefore, during the period between 182 and 273 days, the change of persistent correlation is relatively small. For $[\Delta \tau]$ in the period of 273 to 365 days, the values of $\Delta S$ in the El Nino region further decrease. The values of $C(\tau)$ become closer to the random state, which correspond to the small $[\Delta S]$.

The results for $[\Delta \tau]$ in the period of 365 to 730 days are presented in the other four panels. The signal energy becomes weaker and weaker as the starting time of $\tau_{1}$ increases since the surface temperature is mostly in a random state.

In summary, by considering the portion of $[\Delta \tau]=$ $\tau_{2}-\tau_{1}$, we can remove the information of $C(\tau)$ before $\tau_{1}$ and focus on the specific temporal range of $\tau_{2}-\tau_{1}$. The results show that the change of correlation in the surface temperature happens mostly during the $[\Delta \tau]$ from 90 to 365 days, and this information was not demonstrated in $\Delta S$, as shown in Fig. 3.

\section{Conclusion}

ACF itself provides a measure of the influence of leading fluctuation at the origin at time $t$ on the behavior at a temporal interval $\tau$ apart, which represents the possibility of persistent climate events between two temporal moments. However, this method has never been addressed in climate study. By using the surface screen temperature from ECMWF reanalysis data, it is shown that the climate persistence exists to some extension. For a short time interval of $\tau=5$ days, the eastern part of North America has relatively smaller $C(\tau)$ compared to the western part. Thus, the persistence chance of surface temperature is larger in the west part. The values of $C(\tau)$ over the ocean are very close to one, as the ocean temperature is very stable in a short period. With the increase of $\tau, C(\tau)$ over most of the land quickly approaches zero within one month, the persistent chance of temperature is minimal over the land. In contrast, the persistence for the ocean is much larger, especially in the El Nino regions with strong correlation presented at $\tau=$ 90 days.

Figure 2 shows that the trend of decay for the spatial averaged ACF is noticeable, and by linear fitting to a specific range of $\tau$, the geophysical distributions of the critical exponent display very different results for different $\tau$. The decay of ACF is evident in both land and ocean; however, the power-law decay rule is not true. Also, the ACF enters a random noise state when $\tau$ reaches a certain value. Therefore using the critical exponent, $\alpha$, to study the decay of ACF is invalid. The signal energy represents the frequency accumulated results of Fourier transform to ACF in specific periods. The signal energy has an upper limit of one, and it can filter out the random noise. It is found, for a short period $(\Delta \tau<5$ days) the distributions of the signal energy and critical exponent are very similar, with a correlation coefficient over 0.97 . For a more extended time period, though the approach by ACF decay becomes invalid, the signal energy can always be used as an effective way to investigate the change of the correlation in different lengths of the temporal interval. The longer the period, the larger the signal energy and the less persistence in the surface temperature.

The seasonal results of signal energy are discussed as well. In the boreal winter, the relatively large values of signal energy appear in the southern part of North America (for $\Delta \tau=5$ days), and the result becomes opposite in the boreal summer. Therefore, in North America the surface temperature in a short period is more stable in the winter season compared to the summer season. Interestingly, this kind of result reversal also occurs in the eastern and western parts of the Eurasian Continent.

The method of signal energy can also be applied to a specific range of temporal interval. In different temporal intervals, the signal energies exhibit very different results, especially over the El Nino regions. The information regarding the correlation of surface temperature provided by the signal energy can be over 2 years, which is not available by using the decay of ACF.

ACF has been used in studying the persistence in atmospheric events; however, to our knowledge, the attention has seldom been paid to signal energy. For a finite length of data, the basic frequency is different; thus, the variations in different temporal domains are resolved differently, which is helpful in description of the variations in different temporal length. It is shown, for a longer time period, both $\mathrm{ACF}$ and the decay rate of ACF fall in white noise stage. However, because the signal energy can filter out the white noise, it is valid for any temporal lengths. Our study shows that the method of signal energy is superior to that of ACF in all aspects. 


\section{Appendix . Fluctuation analysis and confidence interval}

\section{A.1. Fluctuation analysis}

We further test the signal energy by applying it to the time series of ECMWF daily data of geopotential height at 500 hPa. The data cover 13880 days (1979/1/1 to 2016/12/31) (Poli et al. 2016). Also the results by ACF and FA are presented.

For a time series $\psi(i)$, the residual in a window of length $n$ is,

$S(n)=\sum_{i=1}^{n}[\psi(i)-\bar{\psi}]=\sum_{i=1}^{n} \hat{\psi}(i)$,

where $\bar{\psi}$ is the time average for the window of $n$, then

$$
\begin{aligned}
\left\langle S^{2}(n)\right\rangle & =\left\langle\sum_{i=1}^{n} \hat{\psi}^{2}(i)\right\rangle+\left\langle\sum_{i \neq j}^{i, j \leq n} \hat{\psi}(i) \hat{\psi}(j)\right\rangle \\
& =\left\langle\sum_{i=1}^{n} \hat{\psi}^{2}(i)\right\rangle+\sum_{i \neq j}^{i, j \leq n} C(|i-j|) \\
& =n \overline{\hat{\psi}^{2}}+2 \sum_{k=1}^{n-1}(n-k) C(k)
\end{aligned}
$$

The FA function is defined (Hurst 1951)

$\operatorname{FA}(n)=\sqrt{\left\langle S^{2}(n)\right\rangle}$.

Equation (A.1.3) shows that the main part of FA is a summation of $C(k)$. If the power-law scalings of $C(k) \sim$ $k^{-\alpha}$ and $\mathrm{FA}(n) \sim n^{H}$ are held, under the condition $n \rightarrow \infty$, (Hurst 1951) proved $H=1-\alpha / 2$. For the power-law of $C(k) \sim k^{-\alpha}$, the increase of temporal interval, $\tau$ corresponds to the decrease of autocorrelation. For the power-law of $\mathrm{FA}(n) \sim n^{H}$, the increase in window width, $n$ corresponds the increase of residual. With the trend removal, FA is evolved to DFA. By diving the whole window into a number of sections, and $S(n)$ becomes,

$S(n)=\sum_{i=1}^{n}\left[\psi(i)-\overline{\psi_{m}}\right]$

where $\overline{\psi_{m}}$ is domain mean (or polynomial fit) of a section, and the temporal point, $i$ is located. Generally, DFA can produce a more accurate result of the power-law slope. In the following calculations, the results of FA and DFA are very similar, since both of the trends of climate change and annual cycle have been removed in the input data.

The top panel of Fig. 6 shows the results of ACF for the global mean, tropical mean $\left(23^{\circ} \mathrm{S}-23^{\circ} \mathrm{N}\right)$, northern
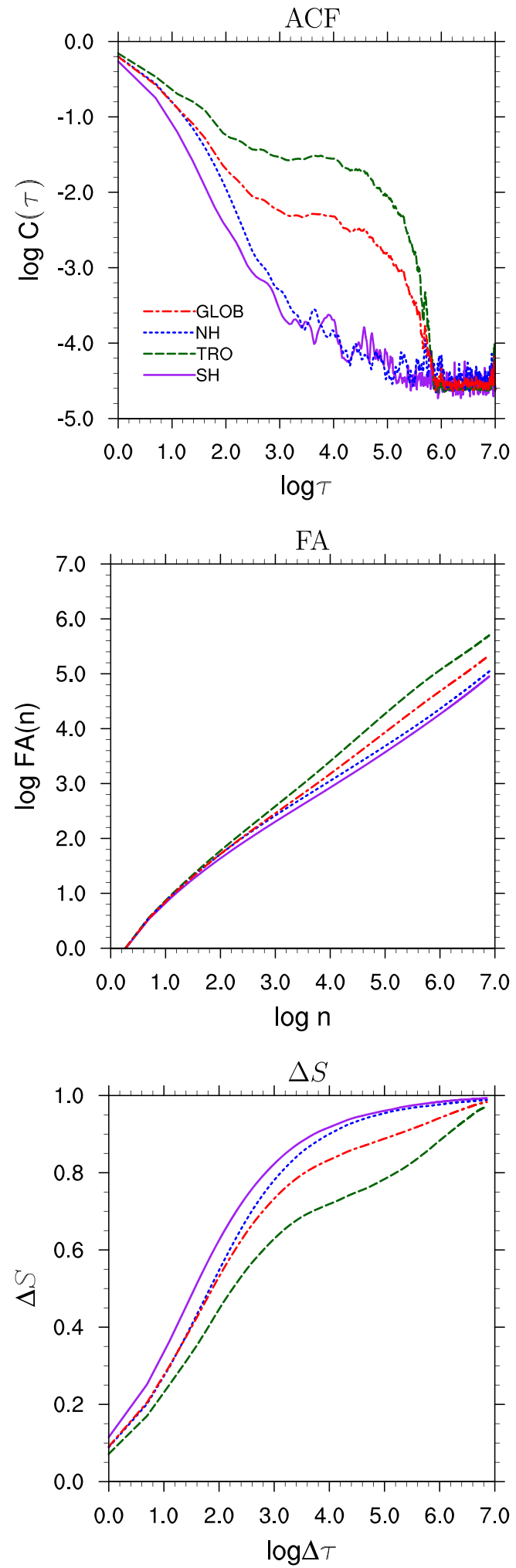

Fig. 6 Based on daily mean geopotential height at $500 \mathrm{hPa}$ from $1979 / 1 / 1$ to $2016 / 12 / 31$, the top panel shows the results of ACF for global mean (red), tropical mean (green), northern hemispheric mean (blue) and southern hemispheric mean (purple), the middle and bottom panels show the corresponding results of FA and $\Delta S . \tau, n$ and $\Delta \tau$ are in the unit of the day 
and southern hemispheric means $\left(23^{\circ}-90^{\circ} \mathrm{N}, 23^{\circ}-90^{\circ} \mathrm{S}\right)$. No curve follows the power-law scaling. It is seen that the decay of the correlations in tropics is slower than those of northern and southern hemispheres, because the tropical climate variability is smaller compared to the extra-tropics. In the tropics, the difference between any consequent days is relatively small, and climate persistence is strong. The middle panel shows the result of FA, the power-law scaling holds for all cases. Since the smaller the slope, the stronger the persistence, the results of FA are consistent with ACF. The constant Hurst exponential, $H$, of FA (DFA) is caused by the cancellation of the noise due to of the summation of $C(k)$ as shown in Eq. (A.1.2).

The bottom panel shows the results of signal energy. The curves become much smoother compared to ACF as the noise of ACF has been filtered out. The differences between each curve become much clear compared to those of FA. It is shown that the curve of tropics approaches to the saturation state of $\Delta S=1$ slowly compared the curves of northern and southern hemispheres. Near $\log \tau=3.2$, the result of ACF is nonphysical, as an increase of $C(\tau)$, which means that the autocorrelation becomes stronger with an increase of temporal interval. However, this noise has been filtered out in signal energy, because $\Delta S$ continue approaching the saturation state. When $\log \Delta \tau>4$ ( $\Delta \tau$ about 54 days), the results of $\Delta S$ for northern and southern hemispheres are close to 1 (saturated) because the corresponding curves of $C(\tau)$ are in the random noisy state. This type of function is not available in FA (DFA).

$\Delta S$ is superior to FA, FA provides only one slope for the whole window, but $\Delta S$ shows the changing persistence at different stages. The saturation of $\Delta S$ provides a criterion for the ultimate loss of system persistence.

\section{A.2. Confident interval}

In statistics, a confidence interval is a type of interval estimate for the mean. Interval estimates are often required since the estimate of the mean varies from sample to sample. The confidence interval provides a lower and upper limits for the mean. The narrower the confidence interval, the deviation to the mean result is smaller.

Since $C(\tau)=\langle\hat{\psi}(t) \hat{\psi}(t+\tau)\rangle$, we can consider $\hat{\psi}(t)$ and $\hat{\psi}(t+\tau)$ are a pair of time series. $r=C(\tau)$ is the correlation coefficient. We first perform a Fisher transformation to $r$, which is an approximate variance-stabilizing transformation for $\mathrm{r}$. After Fisher transformation, the the variance becomes approximately constant for all values of the population correlation coefficient. Thus, the confidence interval can be calculated, since the confidence interval is dependent on variance (or standard deviation).
Following (Bonett and Wright 2000) and (Schweder and Hjort 2016), by Fisher transformation,

$z_{r}=\frac{1}{2} \ln \left(\frac{1+r}{1-r}\right)$

The upper and lower confidence limits can be calculated by $z_{U}$ and $z_{L}$ as

$z_{U} / z_{L}=z_{r} \pm z_{1-\alpha / 2} \sqrt{\frac{1}{N-3}}$

where $\alpha$ is the confidence level and $N$ is the length of the data. The confidence interval is

$W=\frac{\exp 2 z_{U}-1}{\exp 2 z_{U}+1}-\frac{\exp 2 z_{L}-1}{\exp 2 z_{L}+1}$

Equation (A.2.3) show that the confidence interval depends on three factors: the selected confidence level, the values of the correlation coefficients, and the length of the dataset. Note that the wider the confidence interval, the lower the likelihood of reaching the selected confidence level. For example, if the confidence level is set to 0.9 , then the result has $90 \%$ probability to appear in the range of (mean -0.5 interval, mean +0.5 interval).

Open Access This article is licensed under a Creative Commons Attribution 4.0 International License, which permits use, sharing, adaptation, distribution and reproduction in any medium or format, as long as you give appropriate credit to the original author(s) and the source, provide a link to the Creative Commons licence, and indicate if changes were made. The images or other third party material in this article are included in the article's Creative Commons licence, unless indicated otherwise in a credit line to the material. If material is not included in the article's Creative Commons licence and your intended use is not permitted by statutory regulation or exceeds the permitted use, you will need to obtain permission directly from the copyright holder. To view a copy of this licence, visit http:// creativecommonshorg/licenses/by/4.0/.

\section{References}

Bonett D, Wright TA (2000) Sample size requirements for estimating Pearson, Kendall and Spearman correlatios. Psychometrika 65:2328

Chylek P, Tans P, Christy J, Dubey MK (2018) The carbon cycle response to two El Nino types: an observational study. Environ Res Let 13:024001

de Coëtlogon G, Frankignoul C (2003) The persistence of winter sea surface temperature in the north atlantic. J Climate 16:1364-1377

Eichner JF, Koscielny-Bunde E, Bunde1 A, Havlin S, Schellnhuber H$\mathrm{J}$ (2003) Power-law persistence and trends in the atmosphere. Phys Rev E 68:046 133

Fante RL (1988) Signal analysis and estimation. A Wiley Interscience publication, New York

Gerhard E (2000) Sedimentary basins: evolution, facies, and sediment budget. Springeri, 234

He W, Zhao S, Liu Q, Jiang Y, Deng B (2016) Long-range correlation in the drought and flood index from 1470 to 2000 in eastern China. Int J Climatol 36:1676-1685 
Hurst HE (1951) Long-term storage capacity of reservoirs. Am Soc Civil Eng 116:770-799

Kantelhardt JW, Koscielny-Bunde E, Rego HHA, Havlin S, Bunde A (2001) Detecting long-range correlations with detrended fluctuation analysis. Physica 295 A:441-454

Koscielny-Bunde E, Bunde SHA, Goldreich Y (1996) Analysis of daily temperature fluctuations. Physica 231 A:393-396

Koyama J, Hara H (1992) Scaled Langevin equation to describe the $1 / f^{\alpha}$ spectrum. Phys Rev A 46:247-267

Li J, Sun Z (2020) Climate persistence and memory. Tellus A 72:1-16

Lin G, Chena X, Fu Z (2007) Temporal/spatial diversities of longrange correlation for relative humidity over China. Physica A, 383. https://doi.org/10.1016/j.physa.2007.04.059

Lorenz EN (1963) Deterministic nonperiodic flow. J Atmos Sci 20:130-141

Monahan AH (2012) Temporal autocorrelation structure of sea surface winds. J Climate 25:6684-6700

Monettia RA, Havlina S, Bunde A (2003) Long-term persistence in the sea surface temperature fluctuations. Physica A 320:581-589

Peng EACK (1994) Mosaic organization of DNA nucleotides. Phys Rev E 49:1685-1689

Poli P, Hersbach H, Dee DP (2016) ERA-20C: an atmospheric reanalysis of the twentieth century. J Clim 29:4083-4097

Rypdal K, Vand L, Rypdal M (2013) xLong-range memory in Earth's surface temperature on time scales from months to centuries. J Geophys Res, 118. https://doi.org/10.1002/jgrd.50399
Schweder T, Hjort NL (2016) Confidence likelihood. Probability: statistical inference with confidence distributions. Cambridge University Press, Cambridge

Talkner P, Weber RO (2000) Power spectrum and detrended fluctuation analysis: application to daily temperatures. Phys Rev E 62:150-160

Tsonis A, Roebber P, Elsner J (1999) Long-range correlations in the extratropical atmospheric circulation: origins and implications. $\mathbf{J}$ Climate 12:1534-1541

Vyushin D, Kushner P (2009) Power-law and long-memory characteristics of the atmospheric general circulation. J Climate 22:28902904

Weber RO, Talkner P (2001) Spectra and correlations of climate data from days to decades. Climate and Dynamic, 16. https://doi.org/ 10.1029/2001JD000548

Yuan N, Ding M, Huang Y, et al. (2015) On the long-term climate memory in the surface air temperature records over Antarctica: a nonnegligible factor for trend evaluation. J Clim 28:59225934

Zhao S, He W (2015) Evaluation of the performance of the Beijing Climate Centre Climate System Model 1.1(m) to simulate precipitation across China based on long-range correlation characteristics, vol 120

Publisher's note Springer Nature remains neutral with regard to jurisdictional claims in published maps and institutional affiliations. 